\title{
Simplified perfusion fraction from diffusion- weighted imaging in preoperative prediction of IDH1 mutation in WHO grade II-III gliomas: comparison with dynamic contrast-enhanced and intravoxel incoherent motion MRI
}

\author{
Xiaoqing Wang ${ }^{1}$, Mengqiu Cao1, Hongjin Chen², Jianwei Ge², Shiteng Suo ${ }^{1}$, Yan Zhou' \\ ${ }^{1}$ Department of Radiology, Renji Hospital, School of Medicine, Shanghai Jiao Tong University, Shanghai, China, \\ ${ }^{2}$ Department of Neurosurgery, Renji Hospital, School of Medicine, Shanghai Jiao Tong University, Shanghai, China
}

Radiol Oncol 2020; 54(3): 301-310.

Received 23 March 2020

Accepted 13 May 2020

Correspondence to: Yan Zhou, Department of Radiology, Renji Hospital, School of Medicine, Shanghai Jiao Tong University, 160 Pujian Road, Pudong New District, Shanghai, China. E-mail: clare1475@hotmail.com

Disclosure: No potential conflicts of interest were disclosed.

Background. Effect of isocitrate dehydrogenase 1 (IDHI) mutation in neovascularization might be linked with tissue perfusion in gliomas. At present, the need of injection of contrast agent and the increasing scanning time limit the application of perfusion techniques. We used a simplified intravoxel incoherent motion (IVIM)-derived perfusion fraction (SPF) calculated from diffusion-weighted imaging (DWI) using only three b-values to quantitatively assess IDHI-linked tissue perfusion changes in WHO grade II-III gliomas (LGGs). Additionally, by comparing accuracy with dynamic contrast-enhanced (DCE) and full IVIM MRI, we tried to find the optimal imaging markers to predict IDHI mutation status. Patients and methods. Thirty patients were prospectively examined using DCE and multi-b-value DWI. All parameters were compared between the IDHI mutant and wild-type LGGs using the Mann-Whitney U test, including the DCE MRI-derived $K^{\text {trans, }}, v_{e}$ and $v_{p^{\prime}}$ the conventional apparent diffusion coefficient $\left(A D C_{0,1000}\right)$, IVIM-derived perfusion fraction (f), diffusion coefficient (D) and pseudo-diffusion coefficient $\left(D^{*}\right)$, SPF. We evaluated the diagnostic performance by receiver operating characteristic (ROC) analysis.

Results. Significant differences were detected between WHO grade II-III gliomas for all perfusion and diffusion parameters $(P<0.05)$. When compared to IDHI mutant LGGs, IDHI wild-type LGGs exhibited significantly higher perfusion metrics $(P<0.05)$ and lower diffusion metrics $(P<0.05)$. Among all parameters, SPF showed a higher diagnostic performance (area under the curve 0.861 ), with $94.4 \%$ sensitivity and $75 \%$ specificity.

Conclusions. DWI, DCE and IVIM MRI may noninvasively help discriminate IDHI mutation statuses in LGGs. Specifically, simplified DWI-derived SPF showed a superior diagnostic performance.

Key words: IDHI mutation; glioma perfusion; diffusion-weighted MRI; dynamic contrast-enhanced MRI; intravoxel incoherent motion; 2016 WHO CNS tumor classification

\section{Introduction}

Gliomas, the most common primary intracranial neoplasms in humans, are classified as grade I-IV based on histopathological criteria. Different from grade IV, also known as glioblastoma, the outcome of grade II-III gliomas (lower-grade gliomas, LGGs) are highly variable. Published survival duration of LGGs ranged from 1 to over 15 years, reflecting molecular heterogeneity of these tumors. ${ }^{1-5}$ The 2016 revised fourth edition of the World Health Organization (WHO) classification of tumors of 
the central nervous system defines a large subset of gliomas based on molecular alterations, among which mutation of isocitrate dehydrogenase (IDH1) has shown to be the most important, for this mutation is thought to be a predictor of early steps in gliomagenesis. It has been shown that $70 \%-90 \%$ of LGGs- carry IDH1 mutations, and that IDH1 mutant glioma have a survival benefit associated with the maximal surgical resection, and the use of radiation and chemical therapy. ${ }^{6-8}$ Hence, assessing grade II and III gliomas by genetic alteration, which might be helpful for patient prognosis and clinical treatment, is now a common clinical practice.

The IDH1 gene plays an important role in tumor angiogenesis and vasculogenesis, which have been recognized as hallmarks of histopathological growth and progression of gliomas. ${ }^{9-11}$ Therefore, preoperative assessment of tumor perfusion by MRI may give insight into the IDH1 mutation status, thus aiding in clinical decision making. Several MR perfusion techniques have been developed to evaluate the degree of tissue vascularization. Dynamic contrast-enhanced (DCE) MRI and intravoxel incoherent motion (IVIM) MRI are two common MR perfusion techniques with distinct imaging mechanisms. ${ }^{11-15}$

Using rapid T1-weighted imaging to measure the changes resulting from gadolinium contrast agent leakage in and out of the extracellular extravascular space, DCE MRI enables the determination of several hemodynamic parameters, including the volume transfer constant $\left(K^{\text {trans }}\right)$, the extravascular extracellular volume fraction $\left(v_{\mathrm{e}}\right)$, and the vascular plasma volume fraction $\left(v_{\mathrm{p}}\right) \cdot{ }^{11,16}$ Previous studies have demonstrated the clinical potential of DCE MRI in glioma grading and differential diagnosis. ${ }^{17,18}$ However, the need for an intravenous injection of contrast agent limits its clinical application in patients with renal dysfunction or individuals who are allergic to gadolinium.

IVIM MRI is a variant of conventional diffusionweighted imaging (DWI) in that images at multiple $b$-values are required to fit the two-component mathematical model. In this model, the effect of microcirculation of blood in the capillary network (characterized by the pseudo-diffusion coefficient $\left.D^{*}\right)$ is separated from the pure water diffusion component (characterized by the diffusion coefficient $D$ ). More than eight b-values are typically needed to fully characterize biexponential signal attenuation, thus increasing the scanning time. Some simplified models based on IVIM theory with fewer b-values have been proposed. Both the full and simplified IVIM models have shown their

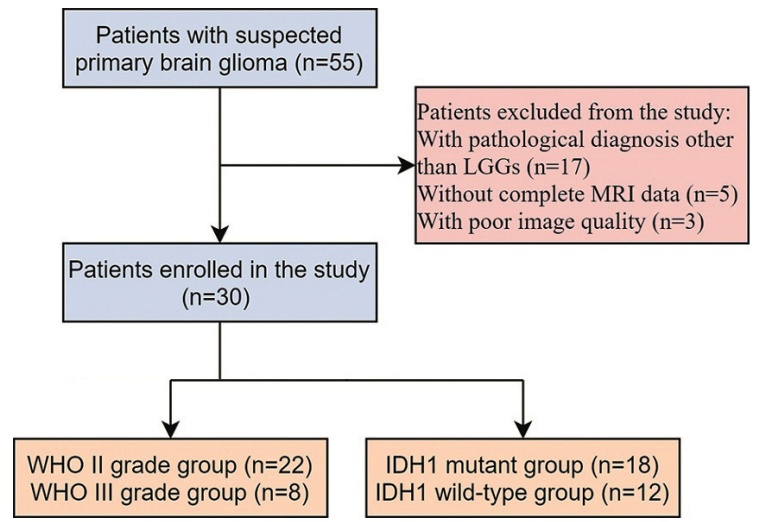

FIGURE 1. Flowchart of study design.

abilities in characterizing tumor perfusion and assessing the glioma grade. ${ }^{19-21}$

The purpose of our study, therefore, was to determine the association of the three b-value DWIderived simplified perfusion fraction $(S P F)$ with tumor perfusion and to compare the performance with DCE and IVIM MRI-derived parameters in the preoperative prediction of IDH1 mutation status in LGGs using surgical and histopathological findings as a standard of reference.

\section{Patients and methods}

\section{Patient enrollment}

This prospective single-center study was performed in accordance with the principle of the Declaration of Helsinki and was approved by the local ethics committee. Written informed consent was obtained from all subjects prior to study enrollment. The flowchart of the study design is demonstrated in Figure 1.

From April 2018 to March 2019, 55 patients who were suspected of primary brain tumors were prospectively enrolled in the study. All patients underwent initial MRI at the same unit and were then underwent neurosurgical resection at our hospital. Excluded from the study were 17 patients with pathological diagnosis other than LGGs, five patients without complete DCE MRI or IVIM data, and three patients due to poor image quality associated with head movement. Finally, a total of 30 patients (13 women, 17 men; average age, 44.73 years; age range, 19-78 years) with histopathologically confirmed LGGs (WHO II glioma, $n=22$; WHO III glioma, $n=8$ ) were enrolled. The descriptive statistics are shown in Table 1. 


\section{MRI acquisition protocols}

MRI of all patients was performed on a 3.0-T MRI unit (Signa HDxt; GE Medical Systems, Milwaukee, WI, USA) using a standard 8-channel head coil. The advanced MRI protocol included DCE MRI and DWI with $10 \mathrm{~b}$-values $\left(0-1000 \mathrm{~s} / \mathrm{mm}^{2}\right)$. Conventional protocol-T1- and T2-weighted imaging with fast spin-echo sequences (T1WI, T2WI), T2 fluid-attenuated inversion recovery (FLAIR) sequence, and contrast-enhanced T1WI- were performed during the same examination.

Three-dimensional DCE MRI of head was performed after intravenous administration of a gadopentetate dimeglumine (Magnevist; Bayer Healthcare, Berlin, Germany, $0.1 \mathrm{mmol}$ per kilogramof body weight) at a rate of $4 \mathrm{ml} / \mathrm{s}$ via a power injector (Spectris; Medrad, Pittsburgh, PA, USA). Precontrast scans with four dynamics were collected before gadopentetate dimeglumine was injected. The detailed parameters of the pre- and postcontrast scans were as follows: repetition time (TR)/echo time (TE), $3.3 \mathrm{~ms} / 1.3 \mathrm{~ms}$, flip angle, $15^{\circ}$; matrix, $256 \times 160$; field of view (FOV), $220 \times 220$ $\mathrm{mm}$; section thickness, $2 \mathrm{~mm}$; number of sections, 40; and total scanning time, $4 \mathrm{~min}$.

DWI was acquired before contrast injection. Ten b-values $(0,20,50,80,150,200,300,500,800$, and $1000 \mathrm{~s} / \mathrm{mm}^{2}$ ) were applied with a fat-suppressed single-shot echo-planar sequence in three orthogonal directions sequentially, they were averaged two times, and then trace images were generated. The other imaging parameters were: TR/TE, 3000 ms/106 ms; matrix, $192 \times 192 ;$ FOV, $260 \times 260$ mm; section thickness/gap, $5 / 1 \mathrm{~mm}$; number of signal averages, 2; number of sections, 15 . The multi-bvalue DWI was acquired at $5 \mathrm{~min}$ and $36 \mathrm{~s}$, and if separately, $2 \mathrm{~min}$ and $11 \mathrm{~s}$ for three-b-value DWI.

\section{MR image analysis}

\section{DCE MRI analysis}

Pharmacokinetic parameters $\left(K^{\text {trans }}, v_{\mathrm{e}^{\prime}} v_{\mathrm{p}}\right)$ were calculated off-line by using commercially available software (MIStar; Apollo Medical Imaging, Melbourne, VIC, Australia) according to the twocompartment Tofts model. ${ }^{22}$ Preprocessing for the perfusion data included semiautomatic selection of arterial input function (AIF). The AIF was obtained independently for every patient from the intracranial internal carotid artery. Parametric maps of $K^{\text {trans, }} v_{\mathrm{e}^{\prime}}$ and $v_{\mathrm{p}}$ were generated on a pixel-by-pixel basis.
TABLE 1. Patient characteristics

\begin{tabular}{|c|c|c|}
\hline Characteristic & $\begin{array}{l}\text { IDH1 mutants } \\
(n=18)\end{array}$ & $\begin{array}{l}\text { IDHI wild-type } \\
\quad(n=12)\end{array}$ \\
\hline Mean age $(y)^{a}$ & $42.8(22-67)$ & 47.9 (19-78) \\
\hline Sex distribution $(M / F)^{b}$ & $10 / 8$ & $7 / 5$ \\
\hline \multicolumn{3}{|l|}{ WHO grade } \\
\hline II & 15 & 7 \\
\hline III & 3 & 5 \\
\hline \multicolumn{3}{|l|}{ Histologic type } \\
\hline Astrocytoma & 12 & 5 \\
\hline Oligodendroglioma & 3 & 0 \\
\hline Oligoastrocytoma & 0 & 1 \\
\hline Anaplastic astrocytoma & 1 & 3 \\
\hline Anaplastic oligodendroglioma & 1 & 2 \\
\hline Anaplastic oligoastrocytoma & 1 & 1 \\
\hline
\end{tabular}

* Mean (range) or count is reported; $a$ = significant difference in age was noted between isocitrate dehydrogenase 1 (IDHI) mutant and wild-type groups $(P=0.020) ; b=$ no significant difference in sex distribution was noted between IDHI mutant and wild-type groups $(P=0.769)$

$F=$ female; $M=$ male

\section{DWI analysis}

DWI data were performed with a program in MATLAB (MATLAB 2017a; MathWorks, Natick, MA, USA) programming tool. Full IVIM features - the diffusion coefficient $(D)$, pseudo-diffusion coefficient $\left(D^{*}\right)$, and the perfusion fraction $(f)$-were extracted by fitting the biexponential model using all b-values as follows:

$$
S_{\mathrm{b}}=S_{0}\left[f_{\exp }\left(-b D^{*}\right)+(1-f) \exp (-b D)\right] \text {, }
$$

where $S_{\mathrm{b}}$ stands for the signal intensity in present b-value and $S_{0}$ stands for the signal intensity in the absence of diffusion gradient.

The monoexponential DWI model used in calculating the $A D C$ value can be written as follows:

$A D C_{\text {low, high }}=-\ln \left(S_{\text {low }} / S_{\text {high }}\right) /\left(b_{\text {low }}-b_{\text {high }}\right)$, where $S_{\text {high }}$ is signal intensity at $b_{\text {high }}$ and $S_{\text {low }}$ is signal intensity at $b_{\text {low' }}$ respectively. As the b-value has a differential sensitivity to Brownian motion of water protons, $A D C_{0,200}$ represents mixed diffusion and perfusion effects and $A D C_{200,1000}$ is almost purely related to diffusion. ${ }^{23,24}$ The b-value scheme was chosen following previous recommendations ${ }^{25-27}$ which indicated that the effects of diffusion and microcapillary perfusion are both reflected within low b-values $\left(b<200 \mathrm{~s} / \mathrm{mm}^{2}\right)$, while for higher $\mathrm{b}$ values $\left(\mathrm{b}>200 \mathrm{~s} / \mathrm{mm}^{2}\right)$, a large proportion of measured signal in each imaging voxel was caused by tissue diffusion. When a typical b-value (1000 s/ $\mathrm{mm}^{2}$ ) was used, the contribution of perfusion has 

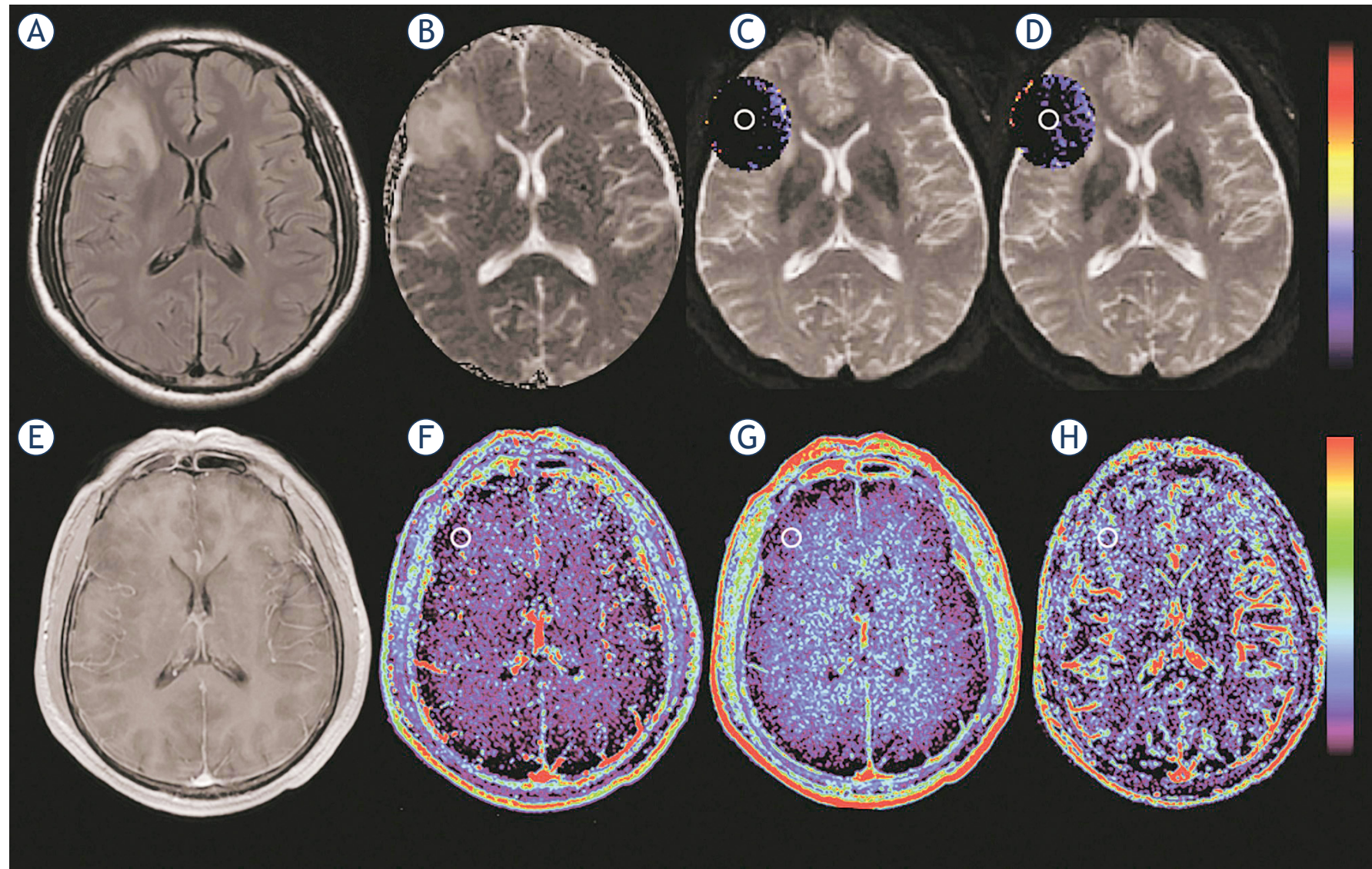

FIGURE 2. Images obtained in a 44-year-old man with astrocytoma (isocitrate dehydrogenase 1 [IDHI] mutant glioma). (A) Fluid-attenuated inversion recovery (FLAIR) image shows a heterogeneous hyperintense lesion in the right frontal lobe. (B) Apparent diffusion coefficient (ADC) 0,1000 map shows increased $A D C$ value in the lesion. (C, D) Intravoxel incoherent motion (IVIM) perfusion fraction ( $f$ ) and simplified perfusion fraction (SPF) maps show no increased values in the corresponding area of the hyperintense lesion as shown in (A). (E) On contrast-enhanced Tl-weighted image, the lesion is non-enhancing. (F-H) Dynamic contrast-enhanced (DCE) MRI parametric maps of volume transfer constant ( $K^{\text {trans }}$ ), extravascular extracellular volume fraction $\left(v_{e}\right)$ and vascular plasma volume fraction $\left(v_{p}\right)$ show no increased values in the lesion. Regions of interest are marked on parametric maps.

TABLE 2. Parameters derived from dynamic contrast-enhanced (DCE) MRI and diffusion-weighted imaging (DWI) between WHO grade II and III gliomas

\begin{tabular}{lccc}
\hline Parameter & Grade II & Grade III & $P$-value \\
\hline$K^{\text {trans }}\left(\mathrm{min}^{-1}\right)$ & $0.067 \pm 0.048$ & $0.116 \pm 0.064$ & 0.013 \\
$v_{\mathrm{e}}$ & $0.071 \pm 0.057$ & $0.401 \pm 0.344$ & 0.018 \\
$\boldsymbol{v}_{\mathrm{p}}$ & $0.036 \pm 0.020$ & $0.051 \pm 0.018$ & 0.035 \\
$\mathbf{A D C} \boldsymbol{C}_{0,1000}\left(\times 10^{-3} \mathrm{~mm}^{2} / \mathrm{s}\right)$ & $1.093 \pm 0.203$ & $0.904 \pm 0.184$ & 0.028 \\
$S P F(\%)$ & $10.78 \pm 4.378$ & $16.391 \pm 5.471$ & 0.012 \\
$D\left(\times 10^{-3} \mathrm{~mm}^{2} / \mathrm{s}\right)$ & $1.194 \pm 0.261$ & $0.949 \pm 0.169$ & 0.021 \\
$D^{*}\left(\times 10^{-3} \mathrm{~mm}^{2} / \mathrm{s}\right)$ & $6.692 \pm 1.564$ & $8.618 \pm 2.215$ & 0.037 \\
$\boldsymbol{f}(\%)$ & $3.315 \pm 1.536$ & $6.380 \pm 3.419$ & 0.020 \\
\hline
\end{tabular}

* $P$-values are considered significant at $P<0.05$

$A D C=$ apparent diffusion coefficient; $D=$ diffusion coefficient; $D^{*}=$ pseudo-diffusion coefficient $f=$ perfusion fraction; $K^{\text {trans }}=$ volume transfer constant; $v_{0}=$ extravascular extracellular volume fraction; $v_{p}=$ vascular plasma volume fraction; $S P F=$ simplified perfusion fraction faded away entirely. The $A D C$ thus appears to be a sensitive index of diffusion component. On the other hand, since a b-value of $1000 \mathrm{~s} / \mathrm{mm}^{2}$ is small enough, high image quality may be guaranteed and the kurtosis effect may be avoided. As the contribution of kurtosis is greater when b-value is beyond $1000 \mathrm{~s} / \mathrm{mm}^{2} .{ }^{28,29}$ Therefore, the relative proportion of the perfusion component in the whole diffusion pool, named SPF, can be determined as follows (20):

$$
S P F=\left(A D C_{0,200}-A D C_{200,1000}\right) / A D C_{0,200} .
$$

\section{Region of interest analysis}

The regions of interest (ROIs) were drawn by two readers who have 6(M.C.) and 19(Y.Z.) years of experience in neuroradiology, respectively, and con- 


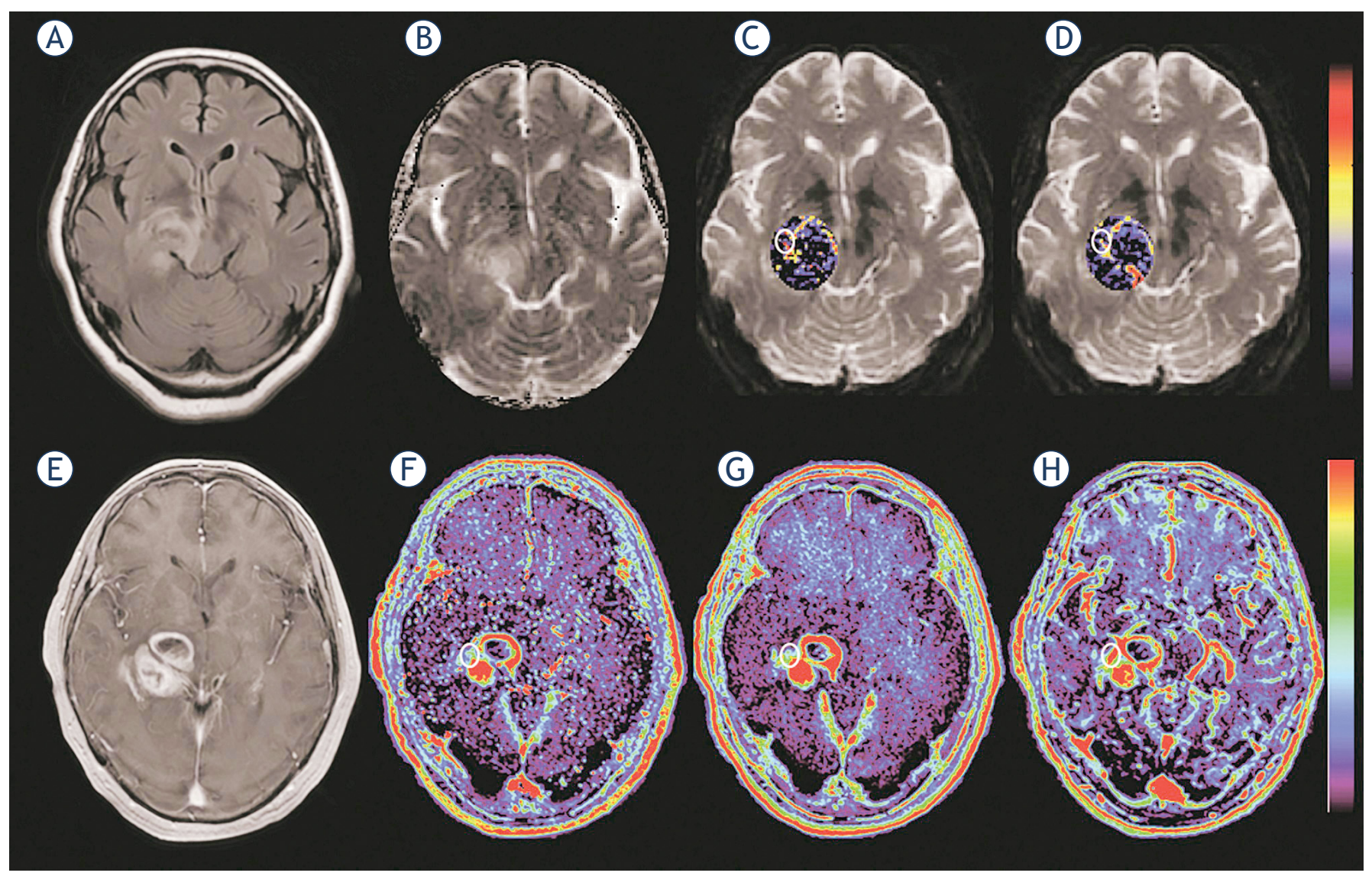

FIGURE 3. Images obtained in a 72-year-old woman with astrocytoma (isocitrate dehydrogenase 1 [IDHI] wildtype glioma). (A) FLAIR shows a heterogeneous hyperintense lesion in the right hemisphere. (B) Apparent diffusion coefficient $(A D C)_{0,1000}$ map shows a mixed pattern of high and intermediate ADC values in the lesion. (C, D) Intravoxel incoherent motion (IVIM) perfusion fraction (f) and simplified perfusion fraction (SPF) maps show markedly increased $f$ and SPF values in the corresponding area of the contrast-enhanced lesion as shown in (E). (E) On contrast-enhanced Tl-weighted image, the lesion is vividly enhanced. ( $F-H)$ Dynamic contrast-enhanced (DCE) MRI parametric maps of volume transfer constant (Krans), extravascular extracellular volume fraction $\left(v_{e}\right)$ and vascular plasma volume fraction $\left(v_{p}\right)$ show obviously increased values in the corresponding area of the contrastenhanced lesion. Regions of interest are marked on parametric maps.

sensus was researched. Both readers were blinded to the histopathological results and other clinical data, including age and gender. Following previous studies ${ }^{30,31}$, an elliptical ROI $\left(20-340 \mathrm{~mm}^{2}\right)$ was placed by each doctor on parametric maps of the solid tumor area as much as possible to include the portion with the minimum values of diffusion (D and $A D C_{0,1000}$ ) and maximum values of perfusion $\left(S P F, f, D^{*}, K^{\text {trans }}, v_{p^{\prime}}\right.$ and $\left.v_{\mathrm{e}}\right)$. For correlation analysis between SPF and other perfusion parameters, the similar-sized ROIs used for SPF images were placed in the corresponding area of DCE images and IVIM images. T1-weighted contrast-enhanced images where contrast agent leakage in tumors was observed were used as a reference to define the ROIs on parametric maps. ${ }^{32,33}$ The study used $A D C$ images combined with T1-weighted, T2-weighted, and FLAIR images to determine the ROI of tumor
TABLE 3. Parameters derived from dynamic contrast-enhanced (DCE) MRI and diffusion-weighted imaging (DWI) between isocitrate dehydrogenase 1 (IDHI) mutant and wild-type gliomas

\begin{tabular}{lccc}
\hline Parameter & IDH1 mutant & IDH1 wild-type & $P$-value* \\
\hline$K^{\text {trans }}\left(\mathrm{min}^{-1}\right)$ & $0.054 \pm 0.024$ & $0.123 \pm 0.073$ & 0.007 \\
$v_{e}$ & $0.052 \pm 0.035$ & $0.121 \pm 0.080$ & 0.007 \\
$v_{\mathrm{p}}$ & $0.032 \pm 0.015$ & $0.051 \pm 0.022$ & 0.015 \\
$A D C_{0,1000}\left(\times 10^{-3} \mathrm{~mm}^{2} / \mathrm{s}\right)$ & $1.123 \pm 0.185$ & $0.923 \pm 0.199$ & 0.009 \\
$S P F(\%)$ & $9.572 \pm 3.437$ & $16.332 \pm 4.925$ & $<0.001$ \\
$D\left(\times 10^{-3} \mathrm{~mm}^{2} / \mathrm{s}\right)$ & $1.108 \pm 0.245$ & $0.959 \pm 0.146$ & 0.047 \\
$D^{*}\left(\times 10^{-3} \mathrm{~mm}^{2} / \mathrm{s}\right)$ & $6.546 \pm 1.757$ & $8.196 \pm 1.794$ & 0.020 \\
$f(\%)$ & $3.080 \pm 1.581$ & $5.712 \pm 2.924$ & 0.005 \\
\hline
\end{tabular}

* $P$-values are considered significant at $P<0.05$

$A D C=$ apparent diffusion coefficient; $D=$ diffusion coefficient; $D^{*}=$ pseudo-diffusion coefficient; $K^{\text {trans }}=$ volume transfer constant; $f=$ perfusion fraction; $S P F=$ simplified perfusion fraction; $v_{e}=$ extravascular extracellular volume fraction; $v_{0}=$ vascular plasma volume fraction 

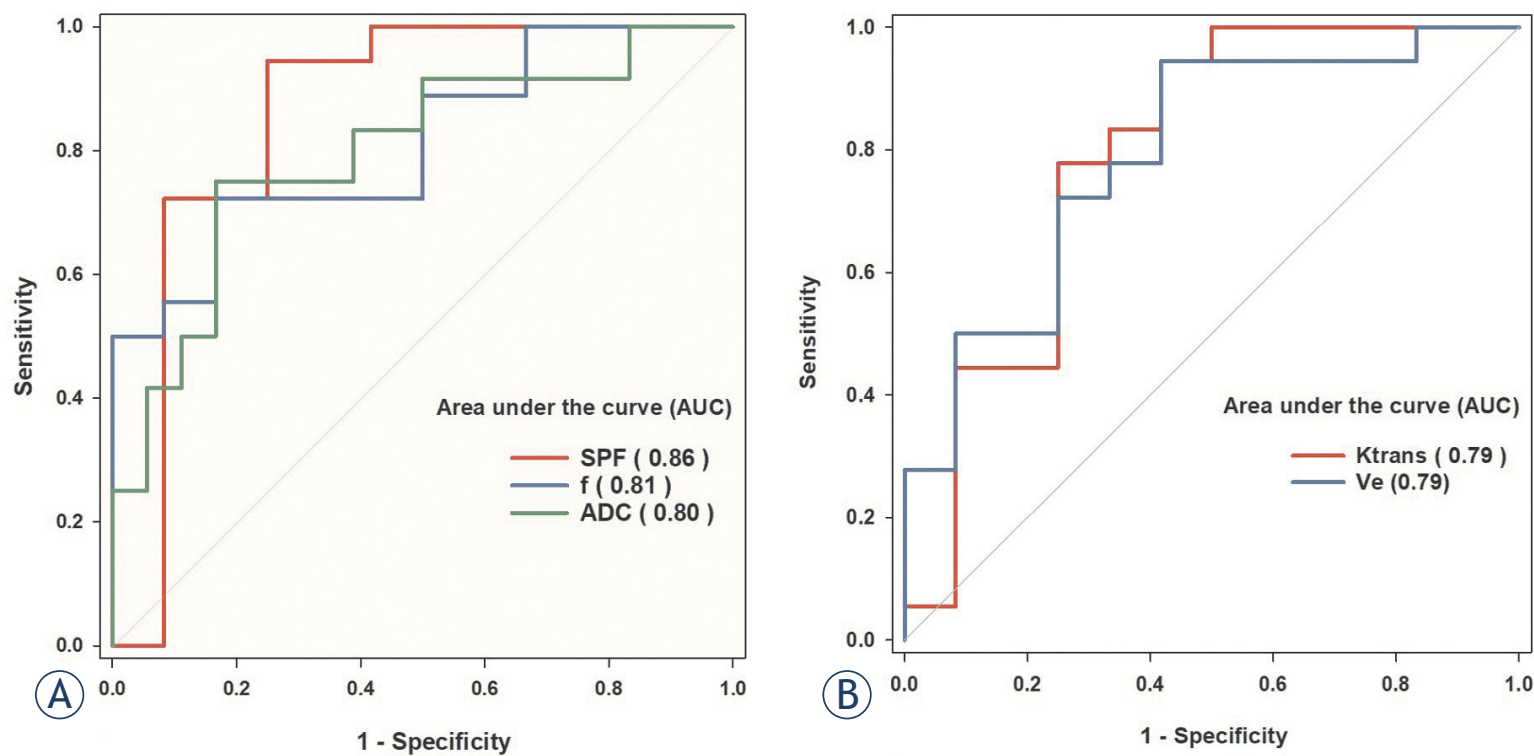

FIGURE 4. Receiver operating characteristic (ROC) curves and corresponding area under the curve values for (A) diffusionweighted imaging (DWI) parameters (simplified perfusion fraction [SPF], perfusion fraction [f], apparent diffusion coefficient $[A D C]_{0,1000}$ ) and (B) dynamic contrast-enhanced (DCE) MRI parameters (transfer constant [ $K^{\text {trans }}$, extravascular extracellular volume fraction $\left[V_{e}\right]$ and vascular plasma volume fraction $\left[V_{p}\right]$ ) in the differentiation of isocitrate dehydrogenase 1 (IDHI) mutant and wildtype gliomas. SPF showed the highest diagnostic performance with the area under the curve value of 0.86 .

area in nonenhancing lesion. Special care was taken to exclude necrosis, cysts, hemorrhage, calcification, and intralesional macrovessels.

\section{Statistical analysis}

Statistical analysis was performed using commercial software (SPSS version 22, IBM Corporation, Armonk, NY, USA and MedCalc, version 11.4.2.0, MedCalc Software, Mariakerke, Belgium). The relationship between perfusion parameters was analyzed with Spearman rank correlation. We considered correlation coefficients $<0.4,0.4-0.6$, $0.6-0.8$, and $>0.8$ to indicate week, moderate, strong, and very strong correlation, respectively. The unpaired t-test and Mann-Whitney $U$ test were used to determine the difference in DWI, DCE and IVIM MRI parameters between WHO grade II and III gliomas, as well as between IDH1 mutant and wild-type gliomas, according to the data normality (Kolmogorov-Smirnov test). ROC curves were constructed to evaluate the ability to identify different IDH1 mutation statuses. Area under the curve (AUC) values of $<0.7,0.7-0.9$, and

TABLE 4. Diagnostic performance of parameters for differentiation between isocitrate dehydrogenase 1 (IDHI) mutant and wildtype gliomas

\begin{tabular}{lcccc}
\hline Parameter & AUC $(95 \% \mathrm{Cl})$ & Sensitivity (\%) & Specificity (\%) & Cutoff value \\
\hline$K^{\text {trans }}\left(\mathrm{min}^{-1}\right)$ & $0.773(0.563-0.983)$ & 77.8 & 75.0 & $>0.062$ \\
$v_{\mathrm{e}}$ & $0.760(0.569-0.951)$ & 94.4 & 58.3 & $>0.119$ \\
$v_{\mathrm{p}}$ & $0.680(0.451-0.909)$ & 55.6 & 91.7 & $>0.029$ \\
$A D C_{0,1000}\left(\times 10^{-3} \mathrm{~mm}^{2} / \mathrm{s}\right)$ & $0.718(0.531-0.904)$ & 83.3 & 75.0 & $\leq 1.002$ \\
$S P F(\%)$ & $0.861(0.686-0.959)$ & 94.4 & 75.0 & $>14.500$ \\
$D\left(\times 10^{-3} \mathrm{~mm}^{2} / \mathrm{s}\right)$ & $0.727(0.541-0.913)$ & 72.2 & 83.3 & $>1.065$ \\
$D^{*}\left(\times 10^{-3} \mathrm{~mm}^{2} / \mathrm{s}\right)$ & $0.690(0.493-0.886)$ & 44.4 & 91.4 & $\leq 5.959$ \\
$f(\%)$ & $0.810(0.658-0.963)$ & 72.2 & 83.3 & $>3.617$ \\
\hline
\end{tabular}

$A D C=$ apparent diffusion coefficient; $D=$ diffusion coefficient; $D^{*}=$ pseudo-diffusion coefficient; $f$ = perfusion fraction; ${ }^{*} K^{\text {trans }}=$ volume transfer constant; $S P F=$ simplified perfusion fraction; $v_{e}=$ extravascular extracellular volume fraction; $v_{p}=$ vascular plasma volume fraction 
$>0.9$ were considered to indicate low, medium, and high diagnostic performance, respectively. Differences between AUC values were analyzed by using the Delong method (34). Optimal thresholds were determined by maximizing the Youden index ((specificity + sensitivity) - 1). A $P$-value less than 0.05 was considered to indicate statistical significance.

\section{Results}

In terms of histology, 16 patients had astrocytomas, three had oligodendrogliomas, one had an oligoastrocytoma, four had anaplastic astrocytomas, three had anaplastic oligodendrogliomas, and three had anaplastic oligoastrocytomas. Intercorrelation analysis between perfusion parameters revealed a significant association for SPF and $f(\varrho=0.768, P$ $<0.001)$. The study also found a moderate correlation between SPF and $v_{\mathrm{e}}(\varrho=0.548, P=0.002)$ and between $S P F$ and $K^{\text {trans }}(\varrho=0.535, P=0.002)$.

The statistical data of DCE MRI and DWIderived parameters in differentiating WHO grade II and III gliomas are summarized in Table 2. Perfusion-related parameters including $K^{\text {trans }}, v_{\mathrm{e}^{\prime}} v_{\mathrm{p}^{\prime}}$ $f, D^{*}$, and $S P F$ were all significantly higher in WHO grade III gliomas than in WHO grade II gliomas (all $P<0.05$ ), while $A D C$ and $D$ values were both significantly lower in WHO grade III gliomas (both $P<0.05)$.

Representative cases of IDH1 mutant and wildtype LGGs are shown in Figures 2 and 3. The mean values \pm standard deviations of DCE MRI and DWI-derived parameters for the IDH1 mutant and wild-type tumors in the whole LGGs group, are summarized in Table 3. Compared with IDH1 mutant LGGs, IDH1 wild-type LGGs exhibited significantly higher perfusion values, that is, $K^{\text {trans }}, v_{\mathrm{e}^{\prime}}$ $v_{p^{\prime}} f, D^{*}$, and SPF (all $P<0.05$ ), and significantly lower diffusion values, that is, $A D C$ and $D$ (both $P$ $<0.05)$. In the WHO grade II subgroup, $v_{\mathrm{p}}$ and $S P F$ differed significantly between IDH1 mutant and wild-type tumors $(P=0.018$ and $P=0.049$, respectively), whereas in the WHO grade III subgroup, only $f$ showed a significant difference $(P=0.014)$.

The results of ROC curve analysis are presented in Figure 4 and Table 4. For differentiation between IDH1 mutant and wild-type LGGs, the ROC curve analysis showed that among all parameters, SPF gave the highest AUC value (0.86), followed by $f$ $(0.81)$ and $A D C(0.80)$, though no significant difference in AUC values was found $(P>0.05)$. The optimal SPF threshold for IDH1 mutation discrimi- nation was $14.5 \%$, with a sensitivity and specificity of $94.4 \%$ and $75.0 \%$, respectively.

\section{Discussion}

In this study, an analysis of DWI, DCE, and IVIM MRI was performed to evaluate the tissue diffusion and perfusion characteristics to identify histological and molecular profiles of LGGs. Our results showed that diffusion and perfusion metrics exhibited substantial differences between WHO grade II and III gliomas, as well as between IDH1 mutant and wild-type LGGs. Among all parameters, the simplified DWI-derived perfusion fraction showed higher efficacy in IDH1 mutation detection, indicating that this recently developed three-b-value DWI approach may serve as a surrogate method for LGGs molecular diagnosis.

DWI, DCE, and IVIM MRI-derived parameters showed significant differences between grade II and III gliomas. Diffusion-related parameters, including $A D C$ and $D$ values, were significantly lower in WHO grade III gliomas; this result is in line with those of previous studies. ${ }^{19,35}$ It is now well established that $A D C$ is strongly correlated with cellularity and the nuclear cytoplasmic ratio in tumor tissue $^{36-38}$, both of which are important criteria in the histopathological grading of gliomas.

Notably, perfusion-related parameters, especially $S P F, f$, and $K^{\text {trans }}$, showed a relatively good performance for glioma grading compared with diffusion parameters. This is most likely due to the increased perfusion feature in higher grade gliomas; $K^{\text {trans }}$ reflects the volume transfer constant of a contrast agent from the plasma space to the extravascular extracellular space. ${ }^{39,40}$ In higher-grade gliomas, active angiogenesis and incomplete basement membrane of tumor neovasculature lead to an increment in microvascular permeability, thus a high $K^{\text {trans }}$ value. A previous study ${ }^{13}$ showed that SPF and IVIM-derived $f$ correlated well with DCE MRI-derived $K^{\text {trans }}$ and were useful in differentiating high- from low-grade gliomas. Our results further show that $f$ and SPF also exhibited significant differences between WHO grade II and III gliomas.

Over the last decade, studies have shown that gliomas with IDH mutation are less aggressive and more sensitive to chemotherapy, contributing to a longer overall survival. ${ }^{41-44}$ Therefore, $I D H$ plays a key role in the determination of the glioma molecular phenotype. Zhao et al. ${ }^{45}$ have shown that compared with IDH1 mutant gliomas, IDH1 wild-type gliomas are characterized by increased 
neoangiogenesis and a higher nuclear cytoplasmic ratio due to the infiltrative nature. Higher vascular proliferation leads to stronger perfusion effects. In this study, DWI, DCE, and IVIM MRI-derived perfusion parameters all showed significant differences between IDH1 mutant and wild-type LGGs. Elevated perfusion was observed in IDH1 wildtype LGGs, which is in agreement with several previous reports using other perfusion imaging techniques. ${ }^{46-48}$ For example, Kickingereder et al. ${ }^{46}$ and Brendle et al. ${ }^{48}$ performed dynamic susceptibility contrast and arterial spin labeling perfusionweighted imaging on patients with LGGs, respectively, and both found significantly higher cerebral blood flow values in IDH1 wild-type LGGs. This could be explained by considering the molecular function of IDH1. Cui et al. ${ }^{49}$ and Reis et al. ${ }^{50}$ suggested that IDH1 mutation is associated with decreased invasiveness and reduced angiogenesis via downregulation of the $\mathrm{Wnt} / \beta$-catenin signaling pathway. Furthermore, the accumulation of 2-hydroxyglutarate, an oncometabolite produced upon IDH1 mutation, has been shown to affect hypoxiainducible factor (HIF) levels and the HIF response and may, consequently, reduce hypoxia-induced neovascularization. ${ }^{51}$

According to our ROC curve analysis, the simplified DWI-derived perfusion fraction showed a superior diagnostic accuracy as a predictor for IDH1 mutation in LGGs compared to the full IVIM-derived $f$. This result suggests that the threeb-value simplified DWI approach could save substantial scanning time compared with the full IVIM approach, with no loss of diagnostic efficiency. Additionally, both simplified and full IVIM perfusion performed better than DCE MRI. These two perfusion methods represent different aspects of vasculature. IVIM measures microscopic translational motions associated with microcirculation of blood in the capillary network, while DCE MRI measures capillary leakage of gadolinium contrast agent based on pharmacokinetic modeling. When WHO grade II and III gliomas were analyzed separately, we found SPF exhibited a statistically significant difference in assessing IDH1 mutation status of WHO grade II tumors, whereas $\mathrm{f}$ helped assess WHO grade III tumors. However, these preliminary results must be interpreted with caution due to the small sample size. Besides perfusion, diffusion parameters like $A D C_{0,1000}$ were also predictive of IDH1 mutation in LGGs, with a lower diffusion coefficient found in IDH1 wild-type tumors. Our findings are in agreement with the existing literature regarding their association. ${ }^{47,52}$

Our study has several limitations. First, the cohort was relatively small, especially that of $\mathrm{WHO}$ grade III LGGs $(n=8)$. Therefore, we may have underestimated some associations, such as the association between perfusion-related metrics and IDH1 mutation status, in WHO grade III gliomas. A further prospective study with a larger cohort should be performed to validate our results. Second, estimation bias may occur as a result of different cutoff $b$-values for IVIM analysis. Therefore, the set of $b$-values needs to be further optimized for brain tumors. Finally, the placement of ROIs was subjective and specific to a limited area on MRI. Automatic segmentation and image analysis of the entire tumor volume may improve preoperative risk stratification.

In conclusion, DWI, DCE, and IVIM MRI can be used as quantitative perfusion methods in preoperative IDH1 mutation prediction in LGGs. Specifically, the simplified DWI-derived perfusion fraction showed a superior diagnostic performance, which holds the potential to serve as a contrast-free and time-saving alternative in the clinical setting. However, further validation in a large patient population is warranted.

\section{Acknowledgements}

YZ, STS, MQC, XQW contributed to the conception and design of the study. Data collection and evaluation were carried out by HJC, MQC, XQW as well as JWG. Statistical analyses and visualization were performed by XQW and STS. The manuscript was written by XQW, STS, YZ and MQC. All authors critically reviewed and approved the manuscript.

This work was supported by the National Natural Science Foundation of China [grant numbers 81501458, 81701642, 81571650, and 81901693); Shanghai Science and Technology Committee Medical Guide Project (western medicine) (grant number17411964300); Shanghai Municipal Education Commission-Gaofeng Clinical Medicine Grant Support (grant number 20172013); Medical Engineering Cross Research Foundation of Shanghai Jiao Tong University (grant number YG2015QN37, YG2017QN47) and Incubating Program for Clinical Research and Innovation of Ren Ji Hospital, School of Medicine, Shanghai Jiao Tong University (PYIII-17-027). 


\section{References}

1. Cancer Genome Atlas Research N, Brat DJ, Verhaak RGW, Aldape KD, Yung WKA, Salama SR, et al. Comprehensive, integrative genomic analysis of diffuse lower-grade gliomas. The N Engl J Med 2015; 372: 2481-98. doi: 10.1056/NEJMoa1402121

2. Chang EF, Clark A, Jensen RL, Bernstein M, Guha A, Carrabba G, et al. Multiinstitutional validation of the University of California at San Francisco Low-Grade Glioma Prognostic Scoring System. J Neurosurg 2009; 111: 20310. doi: $10.3171 / 2009$.2.Jns081101

3. Chang EF, Smith JS, Chang SM, Lamborn KR, Prados MD, Butowski N, et al. Preoperative prognostic classification system for hemispheric lowgrade gliomas in adults. J Neurosurg 2008; 109: 817-24. doi: 10.3171/ jns/2008/109/11/0817

4. Karim AB, Maat B, Hatlevoll R, Menten J, Rutten EH, Thomas DG, et al. A randomized trial on dose-response in radiation therapy of low-grade cerebral glioma: European Organization for Research and Treatment of Cancer (EORTC) Study 22844. Int J Radiat Oncol Biol Phys 1996; 36: 549-56. doi: 10.1016/s0360-3016(96)00352-5

5. van den Bent MJ. Practice changing mature results of RTOG study 9802 : another positive PCV trial makes adjuvant chemotherapy part of standard of care in low-grade glioma. Neuro Oncol 2014; 16: 1570-4. doi: 10.1093/ neuonc/nou297

6. Delfanti RL, Piccioni DE, Handwerker J, Bahrami N, Krishnan A, Karunamuni $\mathrm{R}$, et al. Imaging correlates for the 2016 update on WHO classification of grade II/III gliomas: implications for IDH, $1 \mathrm{p} / 19 \mathrm{q}$ and ATRX status. J Neurooncol 2017; 135: 601-9. doi: 10.1007/s11060-017-2613-7

7. Beiko J, Suki D, Hess KR, Fox BD, Cheung V, Cabral M, et al. IDH1 mutant malignant astrocytomas are more amenable to surgical resection and have a survival benefit associated with maximal surgical resection. Neuro Oncol 2014; 16: 81-91. doi: 10.1093/neuonc/not159

8. Villani V, Merola R, Vidiri A, Fabi A, Carosi M, Giannarelli D, et al. Temozolomide low-dose chemotherapy in newly diagnosed low-grade gliomas: activity, safety, and long-term follow-up. Tumori 2017; 103: 255-60. doi: $10.5301 /$ tj.5000565

9. Carmeliet $\mathrm{P}$, Jain RK. Molecular mechanisms and clinical applications of angiogenesis. Nature 2011; 473: 298-307. doi: 10.1038/nature10144

10. Onishi M, Ichikawa T, Kurozumi K, Date I. Angiogenesis and invasion in glioma. Brain Tumor Pathol 2011; 28: 13-24. doi: 10.1007/s10014-010-0007-z

11. Gaddikeri S, Gaddikeri RS, Tailor T, Anzai Y. Dynamic contrast-enhanced MR imaging in head and neck cancer: techniques and clinical applications. AJNR Am J Neuroradiol 2016; 37: 588-95. doi: 10.3174/ajnr.A4458

12. Roberts HC, Roberts TP, Brasch RC, Dillon WP. Quantitative measurement of microvascular permeability in human brain tumors achieved using dynamic contrast-enhanced MR imaging: correlation with histologic grade. AJNR Am J Neuroradiol 2000; 21: 891-9.

13. Knopp EA, Cha S, Johnson G, Mazumdar A, Golfinos JG, Zagzag D, et al. Glial neoplasms: Dynamic Contrast-enhanced T2*-weighted MR imaging. Neuroradiology 1999; 211: 791-8. doi: 10.1148/radiology.211.3.r99jn46791.

14. Hino T, Togao O, Hiwatashi A, Yamashita K, Kikuchi K, Momosaka D, et al. Clinical efficacy of simplified intravoxel incoherent motion imaging using three b-values for differentiating high- and low-grade gliomas. PLoS One 2018; 13: e0209796-e. doi: 10.1371/journal.pone.0209796

15. Wang X, Chen XZ, Shi L, Dai JP. Glioma grading and IDH1 mutational status: assessment by intravoxel incoherent motion MRI. Clin Radiol 2019; 74: 651. e7-651.e14. doi: 10.1016/j.crad.2019.03.020

16. Koh DM, Collins DJ, Orton MR. Intravoxel incoherent motion in body diffusion-weighted MRI: reality and challenges. AJR Am J Roentgenol 2011; 196: 1351-61. doi: 10.2214/AJR.10.5515

17. Awasthi $R$, Rathore RK, Soni $P$, Sahoo $P$, Awasthi $A$, Husain $N$, et al. Discriminant analysis to classify glioma grading using dynamic contrastenhanced MRI and immunohistochemical markers. Neuroradiology 2012; 54: 205-13. doi: 10.1007/s00234-011-0874-y

18. Lu S, Gao Q, Yu J, Li Y, Cao P, Shi H, et al. Utility of dynamic contrastenhanced magnetic resonance imaging for differentiating glioblastoma, primary central nervous system lymphoma and brain metastatic tumor. Eur J Radiol 2016; 85: 1722-7. doi: 10.1016/j.ejrad.2016.07.005
19. Togao O, Hiwatashi A, Yamashita K, Kikuchi K, Mizoguchi M, Yoshimoto K, et al. Differentiation of high-grade and low-grade diffuse gliomas by intravoxel incoherent motion MR imaging. Neuro Oncol 2016; 18: 132-41. doi: 10.1093/neuonc/nov147

20. Cao M, Suo S, Han X, Jin K, Sun Y, Wang Y, et al. Application of a simplified method for estimating perfusion derived from diffusion-weighted MR imaging in glioma grading. Front Aging Neurosci 2018; 9: 432. doi: 10.3389/ fnagi.2017.00432

21. Conklin J, Heyn C, Roux M, Cerny M, Wintermark M, Federau C. A simplified model for intravoxel incoherent motion perfusion imaging of the brain AJNR Am J Neuroradiol 2016; 37: 2251-7. doi: 10.3174/ajnr.A4929

22. Shukla-Dave A, Lee NY, Jansen JFA, Thaler HT, Stambuk HE, Fury MG, et al. Dynamic contrast-enhanced magnetic resonance imaging as a predictor of outcome in head-and-neck squamous cell carcinoma patients with nodal metastases. Int J Radiat Oncol Biol Phys 2012; 82: 1837-44. doi: 10.1016/j. ijrobp.2011.03.006

23. Thoeny $\mathrm{HC}$, de Keyzer $\mathrm{F}$, Vandecaveye $\mathrm{V}$, Chen $\mathrm{F}$, Sun $\mathrm{X}$, Bosmans $\mathrm{H}$, et al. Effect of vascular targeting agent in rat tumor model: dynamic contrastenhanced versus diffusion-weighted MR imaging. Radiology 2005; 237: 492-9. doi: 10.1148/radiol.2372041638

24. Teruel JR, Goa PE, Sjøbakk TE, Østlie A, Fjøsne HE, Bathen TF. A simplified approach to measure the effect of the microvasculature in diffusion-weighted MR imaging applied to breast tumors: preliminary results. Radiology 2016; 281: 373-81. doi: 10.1148/radiol.2016151630

25. Federau C, Maeder P, O'Brien K, Browaeys P, Meuli R, Hagmann P. Quantitative measurement of brain perfusion with intravoxel incoherent motion MR imaging. Radiology 2012; 265: 874-81. doi: 10.1148/ radiol.12120584

26. Suo S, Cao M, Zhu W, Li L, Li J, Shen F, et al. Stroke assessment with intravoxel incoherent motion diffusion-weighted MRI. NMR Biomed 2016, 29: 320-8. doi: $10.1002 / \mathrm{nbm} .3467$

27. Zhang $Q$, Wang $Y-X$, Ma HT, Yuan J. Cramér-Rao bound for intravoxel incoherent motion diffusion weighted imaging fitting. Conf Proc IEEE Eng Med Biol Soc 2013; 2013: 511-4. doi: 10.1109/EMBC.2013.6609549

28. While PT, Teruel JR, Vidić I, Bathen TF, Goa PE. Relative enhanced diffusivity: noise sensitivity, protocol optimization, and the relation to intravoxe incoherent motion. MAGMA 2018; 31: 425-38. doi: 10.1007/s10334-0170660-x

29. Wu W-C, Chen Y-F, Tseng H-M, Yang S-C, My P-CJER. Caveat of measuring perfusion indexes using intravoxel incoherent motion magnetic resonance imaging in the human brain. Eur Radiol 2015; 25: 2485-92. doi: 10.1007/ s00330-015-3655-x

30. Chung WJ, Kim HS, Kim N, Choi CG, Kim SJ. Recurrent glioblastoma: optimum area under the curve method derived from dynamic contrastenhanced T1-weighted perfusion MR imaging. Radiology 2013; 269: 561-8. doi: 10.1148/radiol.13130016

31. Han X, Suo S, Sun Y, Zu J, Qu J, Zhou Y, et al. Apparent diffusion coefficient measurement in glioma: Influence of region-of-interest determination methods on apparent diffusion coefficient values, interobserver variability, time efficiency, and diagnostic ability. J Magn Reson Imaging 2017; 45: 722 30. doi: 10.1002/jmri.25405

32. Park JE, Kim HS, Park KJ, Kim SJ, Kim JH, Smith SA. Pre-and posttreatment glioma: comparison of amide proton transfer imaging with MR spectroscopy for biomarkers of tumor proliferation. Radiology 2015; 278: 514-23. doi: $10.1148 /$ radiol.2015142979

33. Bisdas S, Braun C, Skardelly M, Schittenhelm J, Teo TH, Thng $\mathrm{CH}$, et al. Correlative assessment of tumor microcirculation using contrast-enhanced perfusion MRI and intravoxel incoherent motion diffusion-weighted MRI: is there a link between them? NMR Biomed 2014; 27: 1184-91. doi: 10.1002/ nbm.3172

34. DeLong ER, DeLong DM, Clarke-Pearson DL. Comparing the areas under two or more correlated receiver operating characteristic curves: a nonparametric approach. Biometrics 1988; 44: 837-45.

35. Zou T, Yu H, Jiang C, Wang X, Jiang S, Rui Q, et al. Differentiating the histologic grades of gliomas preoperatively using amide proton transfer-weighted (APTW) and intravoxel incoherent motion MRI. NMR Biomed 2018; 31. doi: $10.1002 / \mathrm{nbm} .3850$ 
36. Sugahara T, Korogi $Y$, Kochi M, Ikushima I, Shigematu $Y$, Hirai T, et al. Usefulness of diffusion-weighted MRI with echo-planar technique in the evaluation of cellularity in gliomas. J Magn Reson Imaging 1999; 9: 53-60. doi: 10.1002/(sici)1522-2586(199901)9:1<53::Aid-jmri7>3.0.Co;2-2

37. Matsumoto $Y$, Kuroda M, Matsuya R, Kato H, Shibuya K, Oita M, et al. In vitro experimental study of the relationship between the apparent diffusion coefficient and changes in cellularity and cell morphology. Oncol Rep 2009; 22: 641-8. doi: $10.3892 / o r \_00000484$

38. Chen L, Liu M, Bao J, Xia Y, Zhang J, Zhang L, et al. The Correlation between apparent diffusion coefficient and tumor cellularity in patients: a metaanalysis. PLoS One 2013; 8: e79008. doi: 10.1371/journal.pone.0079008

39. Provenzale JM, York G, Moya MG, Parks L, Choma M, Kealey S, et al Correlation of relative permeability and relative cerebral blood volume in high-grade cerebral neoplasms. AJR Am J Roentgenol 2006; 187: 1036-42. doi: 10.2214/AJR.04.0676

40. Mills SJ, Patankar TA, Haroon HA, Balériaux D, Swindell R, Jackson A. Do cerebral blood volume and contrast transfer coefficient predict prognosis in human glioma? AJNR Am J Neuroradiol 2006; 27: 853-8.

41. Houillier C, Wang X, Kaloshi G, Mokhtari K, Guillevin R, Laffaire J, et al. IDH1 or IDH2 mutations predict longer survival and response to temozolomide in low-grade gliomas. Neurology 2010; 75: 1560-6. doi: 10.1212/ WNL.0b013e3181f96282

42. Beiko J, Suki D, Hess KR, Fox BD, Cheung V, Cabral M, et al. IDH1 mutant malignant astrocytomas are more amenable to surgical resection and have a survival benefit associated with maximal surgical resection. Neuro Oncol 2014; 16: 81-91. doi: 10.1212/WNL.0b013e3181f96282

43. Eckel-Passow JE, Lachance DH, Molinaro AM, Walsh KM, Decker PA, Sicotte $\mathrm{H}$, et al. Glioma groups based on $1 \mathrm{p} / 19 \mathrm{q}, \mathrm{IDH}$, and TERT promote mutations in tumors. N Engl J Med 2015; 372: 2499-508. doi: 10.1056/ NEJMoa1407279

44. Cohen AL, Holmen SL, Colman H. IDH1 and IDH2 mutations in gliomas. Curr Neurol Neurosci Rep 2013; 13: 345. doi: 10.1007/s11910-013-0345-4

45. Zhao $S$, Lin $Y, X u$ W, Jiang $W$, Zha Z, Wang $P$, et al. Glioma-derived mutations in IDH1 dominantly inhibit IDH1 catalytic activity and induce HIF-1alpha. Science 2009; 324: 261-5. doi: 10.1126/science.1170944

46. Kickingereder P, Sahm F, Radbruch A, Wick W, Heiland S, Deimling Av, et al. $\mathrm{IDH}$ mutation status is associated with a distinct hypoxia/angiogenesis transcriptome signature which is non-invasively predictable with rCBV imaging in human glioma. Sci Rep 2015; 5: 16238. doi: 10.1038/srep16238

47. Leu K, Ott GA, Lai A, Nghiemphu PL, Pope WB, Yong WH, et al. Perfusion and diffusion MRI signatures in histologic and genetic subtypes of WHO grade II-III diffuse gliomas. J Neurooncol 2017; 134: 177-88. doi: 10.1007/s11060$017-2506-9$

48. Brendle C, Hempel JM, Schittenhelm J, Skardelly M, Tabatabai G, Bender $\mathrm{B}$, et al. Glioma grading and determination of IDH mutation status and ATRX loss by DCE and ASL perfusion. Clin Neuroradiol 2018; 28: 421-8. doi: 10.1007/s00062-017-0590-z

49. Cui D, Ren J, Shi J, Feng L, Wang K, Zeng T, et al. R132H mutation in IDH1 gene reduces proliferation, cell survival and invasion of human glioma by downregulating Wnt/ $\beta$-catenin signaling. Int J Biochem Cell Biol 2016; 73: 72-81. doi: 10.1016/j.biocel.2016.02.007

50. Reis M, Czupalla CJ, Ziegler N, Devraj K, Zinke J, Seidel S, et al. Endothelial $W n t / \beta$-catenin signaling inhibits glioma angiogenesis and normalizes tumor blood vessels by inducing PDGF-B expression. J Exp Med 2012; 209: 1611 27. doi: $10.1084 / \mathrm{jem} .20111580$

51. Koivunen P, Lee S, Duncan CG, Lopez G, Lu G, Ramkissoon S, et al. Transformation by the (R)-enantiomer of 2-hydroxyglutarate linked to EGLN activation. Nature 2012; 483: 484-8. doi: 10.1038/nature10898

52. Villanueva-Meyer JE, Wood MD, Choi BS, Mabray MC, Butowski NA, Tihan T, et al. MRI Features and IDH mutational status of grade II diffuse gliomas: impact on diagnosis and prognosis. AJR Am J Roentgenol 2018; 210: 621-8. doi: 10.2214/AJR.17.18457 